\title{
Self-organization property of Kohonen's map with general type of stimuli distribution
}

\author{
Ali A. Sadeghi \\ Arbeitsgruppe Technomathematik \\ Universität Kaiserslautern \\ 67653 Kaiserslautern,Germany.
}

\begin{abstract}
Here the self-organization property of one-dimensional Kohonen's algorithm in its $2 \mathbf{k}$-neighbor setting with a general type of stimuli distribution and non-increasing learning rate is considered.We prove that the probability of self-organization for all initial values of neurons is uniformly positive. For the special case of a constant learning rate, it implies that the algorithm self-organizes with probability one.
\end{abstract}

Keywords Neural networks, Kohonen's SOM, One-dimensional self-Organization, Winner definition.

\section{Introduction}

Among several models which have been proposed for self-organizing feature maps, the Kohonen algorithm is the most popular one. The analytical behavior of the algorithm and also its very extended areas of application are widely investigated see $[3,5,7,10,11]$ and references therein . Although the algorithm is computationally simple, analytical investigation of its properties turned out to be very complicated. The existing results , including this paper, deal basically with the one-dimensional Kohonen algorithm.

The Kohonen net consists of a layer of neurons which are labelled from 1 to $N$. The set of neurons, denoted here by $I$, has a fixed neighborhood relationship, that is, to every neuron $i$ a set of neighboring neurons $V_{i} \subset I$ is assigned. To connect the set of neurons with data space Q, which is usually a subset of $\mathbb{R}^{m}$, each neuron $i$ is mapped to a weight vector $X_{i} \in Q$. 
Every $v \in Q$ corresponds with the winner neurons $i^{*}(v)$ which satisfy

$$
\left\|X_{i^{*}(v)}-v\right\| \leq\left\|X_{i}-v\right\| \quad \forall i \in I,
$$

where $\|$.$\| denotes the Euclidean norm on \mathbb{R}^{m}$. To avoid confusions we occasionally denote the winner $i^{*}(v)$ corresponding to the weight vector $X$ by $i^{*}(X, v)$.

The adaptation of weight vectors will be carried out by an unsupervised learn process . At the end of the learn process the map $v \in Q \mapsto i^{*}(v) \in\{1, \cdots, N\}$ preserves (in some sense) the topology of the data space. The learning starts with an initial weight vector $X^{0}=\left(X_{1}^{0}, \cdots, X_{N}^{0}\right)^{T}$, which will be updated as follows

$$
X_{i}^{n+1}=X_{i}^{n}+\epsilon_{n} f_{i^{*} i}\left(v_{n+1}-X_{i}^{n}\right) \quad \forall i \in I, \quad n=0,1, \cdots
$$

where $v_{n+1} \in Q$ is an independent random variable distributed identically by some probability distribution $P, \epsilon_{n}$ is the so-called learning rate and $f:\left(i, i^{*}\right) \mapsto f_{i i^{*}} \in[0,1]$ is the neighborhood function. The initial weight vector $X^{0}$ may be chosen at random from $Q^{N}$.

In this paper we consider the Kohonen algorithm in its one-dimensional case, where $Q \subset \mathbb{R}$ and $V_{i}=\{i-1, i, i+1\} \cap I$. Moreover

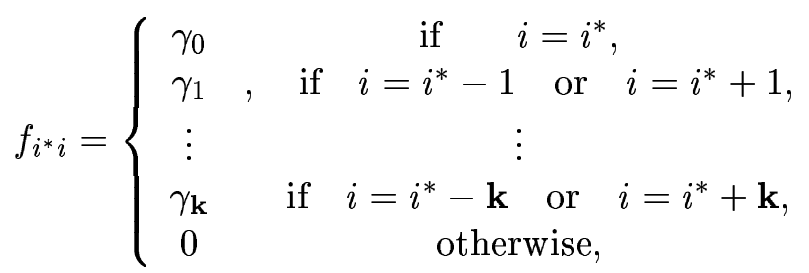

in which $\gamma_{0}=1,0<\gamma_{\mathbf{k}} \leq \cdots \leq \gamma_{1} \leq 1$ and $\mathbf{k}>0$. The probability distribution $P$ is assumed to be diffuse, that is, if $A$ has Lebesgue measure zero, then $P(A)=0$.

The evolution of the weights in the one-dimensional Kohonen algorithm can be decomposed into two phases:

1- Self-organization phase, in which the weights of neurons become organized.The set of organized states for this case reduces to the set of vectors in $\mathbb{R}^{N}$ which have ascending or descending components. An important fact distinguishing the one-dimensional algorithm is , that if the weights become ordered (organized) they retain their ordering for ever.

The existing results concerning the self-organization property of the algorithm are limited to some special cases of the one-dimensional algorithm , see $[1,2,3,4,5,6]$.

To provide a proof of the self-organization, Cottrell and Fort [3] and also Bouton and Pagès $[1,2]$ considered the case that $\epsilon_{n}$ is a constant and the algorithm is a homogeneous Markov process . Using the properties of these kinds of processes, they proved that for a width one neighborhood function ( $\mathbf{k}=1$ with $\gamma_{1}=1$ ) the algorithm self-organizes to a 
topology preserving map with probability one (for both uniform and non-uniform stimuli distributions).

Erwin et al. [4] studied the case of $\mathbf{k} \geq N / 2$ and a uniform stimuli distribution. In the most recent papers Flanagan [5,6] extended the results in [4] to the case of a more general probability distribution of stimuli with the restriction that $\epsilon_{t} \geq \alpha_{m}>0$ and the neighborhood function is restrictly decreasing.

For $\mathbf{k}=0$ it is known that the one-dimensional algorithm fails to make up a topology preserving map.

2- Convergence phase, in which the weight vectors converge to their final values. In this phase learn process (2) reduces to a special case of the Robbins-Monro algorithm and its behavior is governed by the so-called mean (or average) differential equation (m.d.e.). This phase has been studied in $[1,3,7,11]$. Bouton and Pagés [1] considered the one dimensional algorithm in its width one setting with $\gamma_{1}=1$. For uniformly distributed stimuli it was shown that the algorithm converges to the fixed point of the m.d.e. with probability one . Later on Fort and Pagés extended this result to some more general class of neighborhood functions [7].

The almost sure convergence of the algorithm for a quite general class of distributions and neighborhood functions was studied by Sadeghi in [11], where it is shown that the m.d.e. is cooperative and irreducible and the set of organized states is positively invariant. This together with a stability result due to Hirsch implied the almost sure convergence of the algorithm under the condition that the m.d.e. owns a unique fixed point with ordered components.

In this paper we want to extend the knowledge about the algorithm in its self-organization phase. Section 2 includes a generalization of the winner definition, which allows us to investigate the behavior of the algorithm on whole $Q^{N}$. Section 3 includes the main results of the paper, Theorems 1 and 2, where it is shown that regardless of the initial weights of the neurons the probability of self-organization is uniformly positive . We assume no positive lower bound for $\epsilon_{n}$. For the case of a constant learning rate, we show that the self-organization occurs with probability one. The conditions imposed on the neighborhood function are weak enough to ensure the convergence of the algorithm in its quantization phase[11], particularly a big class of non-increasing neighborhood functions is included. Some final remarks are made in section 4.

\section{About the winner}

Without loss of generality in the remainder of the paper we always set $Q=[0,1]$. The following conventions will be adopted :

$$
D:=\left\{x \in[0,1]^{N} \mid x_{k}=x_{l} \Rightarrow k=l \quad \forall \quad k, l \in I\right\}, \quad D^{\prime}:=[0,1]^{N} \backslash D,
$$




$$
\begin{aligned}
F^{+} & :=\left\{x \in[0,1]^{N} \mid \quad 0<x_{1}<x_{2}<\ldots<x_{N}<1\right\}, \\
F^{-} & :=\left\{x \in[0,1]^{N} \mid \quad 1>x_{1}>x_{2}>\ldots>x_{N}>0\right\} \text { and } F:=F^{+} \cup F^{-}, \\
\Psi_{i}^{n} & :=\left\{j \in I \quad \mid \quad X_{i}^{n}=X_{j}^{n}\right\}, \\
S(x, \eta) & :=\left\{y \in[0,1]^{N}|| x_{i}-y_{i} \mid<\eta \quad \forall i \in I\right\} .
\end{aligned}
$$

We denote by $\left|\Psi_{i}^{n}\right|$ the number of elements of $\Psi_{i}^{n}$.

The definition (1) assigns a unique winner neuron for almost all $x \in D$. Moreover, if $x, x^{k} \in D$ and $x^{k} \rightarrow x$, then the Lebesgue measure of the set of stimuli, for which $i^{*}(x, v) \neq i^{*}\left(x^{k}, v\right)$ tends to zero. This assures the stability of the winner on $D$.

To treat the algorithm theoretically, one needs to extend the winner definition, in a way that the uniqueness of the winner neuron on whole $[0,1]^{N}$ is guaranteed. Principally such an extension is easy to do ; many authors have chosen the neuron with the smallest index satisfying (1) as winner for the case of conflict.However, such a definition causes instabilities of winner; the Lebesgue measure of the set of stimuli ,for which $i^{*}(x, v) \neq$ $i^{*}\left(x^{k}, v\right)$ does not tend to zero as $k \rightarrow \infty$. This is source of many difficulties in a mathematical study of the algorithm, see [1]. Sequelly we introduce a new definition of the winner which enables us to overcome the stability problem.

Let $\Sigma_{N}$ be the set of functions $o^{k}($.$) ,$

$$
o^{k}(.):\{1, \cdots, N\} \rightarrow I, \quad k=1, \ldots, N ! .
$$

$\Sigma_{N}$ will be termed in this paper as the set of (inverse) ordering functions ; for $x \in \mathbb{R}^{N}$ let $\Sigma_{N}^{x} \subset \Sigma_{N}$ be the set of those ordering functions $o^{k}($.$) which satisfy$

$$
i>j \Rightarrow x_{o^{k}(i)} \geq x_{o^{k}(j)} \quad \forall i, j \in\{1, \cdots, N\} .
$$

If $x \in D$, then $\Sigma_{N}^{x}$ consists of a unique ordering function which will be called the ordering of the point $x . \quad i$ will be referred as the ordering of the neuron $j$ according to $o^{k}($.$) if o^{k}(i)=j$.

Set

$$
\begin{aligned}
W_{x}^{0}(v) & :=\left\{i \in I \quad|\quad| x_{i}-v|\leq| x_{j}-v \mid \quad \forall \quad j \in I\right\} \\
W_{x}(v) & :=\left\{i \in W_{x}^{0}(v) \quad \mid \quad x_{i} \leq x_{j} \quad \forall \quad j \in W_{x}^{0}(v)\right\} .
\end{aligned}
$$

For any $\quad x \in[0,1]^{N}$ and any given ordering function $o^{k}(.) \in \Sigma_{N}$ we define the $k$-winner $i_{k}^{*}(v)$, or more precisely $i_{k}^{*}(x, v)$, which is in fact a generalization of $i^{*}(v)$ to $[0,1]^{N}$.

DEFINITION. For $i \in W_{x}(v)$ if $v>x_{i} \quad\left(v<x_{i}\right)$, then $i_{k}^{*}(v)$ is the neuron which has the greatest (smallest) ordering in $W_{x}(v)$ according to $o^{k}($.$) .$ 
As a simple example, let $N=3$ and $x=(0.1,0.2,0.2)$. Then

$$
\begin{gathered}
\begin{array}{c}
o^{1}(.)=\{(1,1)(2,2)(3,3)\}, \quad o^{2}(.)=\{(1,1)(2,3)(3,2)\}, \\
o^{3}(.)=\{(1,2)(2,1)(3,3)\}, \quad o^{4}(.)=\{(1,2)(2,3)(3,1)\}, \\
o^{5}(.)=\{(1,3)(2,2)(3,1)\}, \quad o^{6}(.)=\{(1,3)(2,1)(3,2)\},
\end{array} \\
\Sigma_{3}=\left\{o^{k}(.) \quad k=1, \cdots, 6\right\} \text { and } \Sigma_{3}^{x}=\left\{o^{1}(.), o^{2}(.)\right\} . \\
\text { If } v=0.17 \text { then } W_{x}^{0}(v)=W_{x}(v)=\{2,3\}, i_{1}^{*}(v)=i_{3}^{*}(v)=i_{4}^{*}(v)=2 \text { and } i_{2}^{*}(v)= \\
i_{5}^{*}(v)=i_{6}^{*}(v)=3 .
\end{gathered}
$$

It is clear that if $x \in D$, then $W_{x}(v)$ consists of a unique neuron and therefore $i_{k}^{*}(v)=i^{*}(v)$ for almost all $v \in[0,1]$ and all $o^{k}(.) \in \Sigma_{N}$. But if the algorithm starts from a point $x \in D^{\prime}$, then, as soon as $X^{n} \in D^{\prime}$, there exists stimuli $v$ for which $W_{x}(v)$ consists of more than one neuron and consequently the $k$-winner depends on the ordering function $o^{k}($.$) . This means, for x \in D^{\prime}$ the probability that $F$ be reached within $t$ steps depends on the ordering function which we choose. This is in accordance with the fact that in any neighborhood of a point $x \in D^{\prime}$ there exists points of $D$ with all different possible orderings.

Next let us introduce the following function :

$$
P_{x}\left(\tau_{\pi} \leq t\right):=\min _{o^{k}(.) \in \Sigma_{N}^{x}} \mathbf{P}\left(X^{t} \in \pi \mid X^{0}=x \quad ; \quad i^{*}(v):=i_{k}^{*}(v)\right) .
$$

Here $\mathbf{P}\left(. \mid\right.$.) denotes the conditional probability function and $\pi$ is any subset of $[0,1]^{N}$. Note that $P_{x}\left(\tau_{\pi} \leq t\right)$ is also the minimum of $\mathbf{P}\left(X^{t} \in \pi \mid X^{0}=x \quad ; \quad i^{*}(v):=i_{k}^{*}(v)\right)$ over $\Sigma_{N}$.

The rest of this paper deals with the treatment of the analytical properties of $P_{x}\left(\tau_{F} \leq t\right)$.

\section{Self-Organization}

In this section we present a proof of the self-organization property of Kohonen's algorithm. This proof has not only the advantage that it is valid for general $2 \mathbf{k}$-neighbor setting with quite reasonable restrictions on the input distribution and the learning rate, but also it is shorter than the proofs which have been appeared up to now $[1,2,3,4,5]$.

If there exists a $T \in \mathbb{N}$, such that $X^{T} \in F$ with probability one, then the algorithm is said to have the self-organization property.It is known that if $X^{T} \in F$ for some $T$, then $X^{T+n} \in F$ for all $n,[10]$. 
The method we use to establish the self-organization property is to show that the probability of making up an organized configuration within a finite number of steps is uniformly positive for all initial weights. As a first step, in Lemmas $1-3$, we show that $P_{x}\left(\tau_{F} \leq t\right)$ has a positive lower bound if any initial state $x$ can be taken to the organized configuration with a positive probability for any $i_{k}^{*}(v) \in \Sigma_{N}^{x}$. Then, in Lemma 4 and Theorem 1 , we show that this is actually the case.

For $x \in[0,1]^{N}$ suppose $\Sigma_{N}^{x}$ consists of $m$ different ordering functions $o^{i}(),. \quad i=$ $1 \cdots, m, m \leq N$ !. Let $p \in\{1, \cdots, m\}$ and define

$$
\begin{aligned}
& S^{p}(x, \eta):=S(x, \eta) \cap\left\{y \in D \quad \mid \quad \Sigma_{N}^{y}=\left\{o^{p}(.)\right\}\right\}, \quad S^{m+1}(x, \eta):=S(x, \eta) \cap D^{\prime}, \\
& S^{m+1, p}(x, \eta):=\left\{y \in S^{m+1}(x, \eta) \quad \mid \quad P_{y}\left(\tau_{F} \leq t\right)=\mathbf{P}\left(Y^{t} \in F \quad \mid \quad Y^{0}=y, i^{*}(v):=i_{p}^{*}(v)\right)\right\} .
\end{aligned}
$$

For sufficiently small $\eta$ it is easy to verify that $S(x, \eta)=\cup_{p=1}^{m}\left(S^{p}(x, \eta) \cup S^{m+1, p}(x, \eta)\right)$.

$\mathcal{V}_{p}(x)$ is the set of all events $\nu=\left(v_{1}, \cdots, v_{t}\right)$ which take $X^{0}=x$ to $F$ with $i^{*}(v):=$ $i_{p}^{*}(v)$, where $o^{p}(.) \in \Sigma_{N}^{x}$.

Lemma 1. For any $x \in[0,1]^{N}$ and $\nu \in \mathcal{V}_{p}(x)$ there exists a $\eta>0$ such that $\nu$ takes all $y \in S^{p}(x, \eta) \cup S^{m+1, p}(x, \eta)$ to $F$ by putting $i^{*}(v):=i_{p}^{*}(v)$.

Proof. The proof amounts to the study of two different cases.

Case 1, $x \in D$. In this case the winner is uniquely defined and we do not mention explicitly about the ordering function.

Let $\nu=\left(v_{1}, \cdots, v_{n}\right) \in \mathcal{V}_{p}(x)$ be any chain of stimuli which takes $x$ to $F$ and $X^{1}, \cdots, X^{t}$ the corresponding weight vectors. $k$ denotes the ordering of $i^{*}$ at time $n$, that is,$o(k)=i^{*}\left(v_{n}, X^{n}\right)$ and $\bar{X}_{i^{*}}^{n}:=0.5\left(X_{i^{*}}^{n}+X_{o(k-1)}^{n}\right), \quad \bar{X}_{i^{*}+1}^{n}:=0.5\left(X_{i^{*}}^{n}+X_{o(k+1)}^{n}\right)$.

For $x \in D$ there exista a $\eta>0$ such that

$$
\eta<0.5 \min \left(\left|\bar{X}_{i^{*}+1}^{n}-v_{n}\right|,\left|\bar{X}_{i^{*}}^{n}-v_{n}\right|\right), \quad \forall \quad n=0, \cdots, t-1 .
$$

Note that the set of all stimuli for which $\eta$ dose not exist has Lebesgue measure zero. Moreover it is independent of the ordering function $o^{p}($.$) .$

We show, by induction, that if $Y^{0}=y \in S(x, \eta)$, then $Y^{t} \in S\left(X^{t}, \eta\right)$.

Let $Y^{n-1} \in S\left(X^{n-1}, \eta\right)$ for $n \in\{1, \cdots, t\}$. Because of $\eta<0.5 \min \left(\left|\bar{X}_{i^{*}+1}^{n}-v_{n}\right|, \mid \bar{X}_{i^{*}}^{n}-\right.$ $\left.v_{n} \mid\right)$, we have $i^{*}\left(v_{n}, Y^{n-1}\right)=i^{*}\left(v_{n}, X^{n-1}\right)$ and therefore

$$
Y_{i}^{n}=\left(1-\epsilon_{n} \gamma_{\left|i-i^{*}\right|}\right) Y_{i}^{n-1}+\epsilon_{n} \gamma_{\left|i-i^{*}\right|} v_{n} \quad \forall \quad i \in I,
$$


which implies,

$$
X_{i}^{n}-\left(1-\epsilon_{n} \gamma_{\left|i-i^{*}\right|}\right) \eta<Y_{i}^{n}<X_{i}^{n}+\left(1-\epsilon_{n} \gamma_{\left|i-i^{*}\right|}\right) \eta \quad \forall \quad i \in I,
$$

that is, $Y^{n} \in S\left(X^{n}, \eta\right)$.

Recall that $F$ is an open subset of $[0,1]^{N}$, for sufficiently small $\eta$ we have $Y^{t} \in F$.

Case 2, $x \in D^{\prime}$. Consider again any event $\nu=\left(v_{1}, \cdots, v_{n}\right) \in \mathcal{V}_{p}(x)$ and the corresponding weight vectors $X^{1}, \cdots, X^{t}$. Let $k$ be the ordering of $i_{p}^{*}$ at time $n$, that is , o $o^{p}(k)=i_{p}^{*}\left(v_{n}, X^{n}\right)$ and $\bar{X}_{i_{p}^{*}}^{n}:=0.5\left(X_{i_{p}^{*}}^{n}+X_{o^{p}(k-1)}^{n}\right), \quad \bar{X}_{i_{p}^{*}+1}^{n}:=0.5\left(X_{i_{p}^{*}}^{n}+X_{o^{p}(k+1)}^{n}\right)$.

Choose a $\eta>0$ which satisfies

$$
\eta<0.5 \min \left(\left|\bar{X}_{i_{p}^{*}+1}^{n}-v_{n}\right|,\left|\bar{X}_{i_{p}^{*}}^{n}-v_{n}\right|\right), \quad \forall \quad n=0, \cdots, t-1 .
$$

Suppose $Y^{0}=y \in S^{p}(x, \eta) \cup S^{m+1, p}(x, \eta), \quad p \in\{1, \cdots, m\}$. We use induction to show that, after $\nu=\left(v_{1}, \cdots, v_{n}\right)$ with $i^{*}(v):=i_{p}^{*}(v)$, we have $Y^{t} \in S\left(X^{t}, \eta\right)$.

Assume that $Y^{n} \in S\left(X^{n}, \eta\right)$ for all $n \in\{0, \cdots, l-1\}$, where $l \in\{1, \cdots, t-1\}$. We show this is also true for $n=l$.

As a first step let us show that $i_{p}^{*}\left(v_{l}, Y^{l-1}\right)=i_{p}^{*}\left(v_{l}, X^{l-1}\right)$.

It is clear that $i_{p}^{*}\left(v_{1}, Y^{0}\right)=i_{p}^{*}\left(v_{1}, X^{0}\right)$. Now let us assume $i_{p}^{*}\left(v_{n}, Y^{n-1}\right)=i_{p}^{*}\left(v_{n}, X^{n-1}\right)$ for $n=1, \cdots, s-1, \quad s \leq l$. It is enough to show this is also true for $n=s$.

Suppose $\left|\Psi_{i_{p}^{*}\left(v_{s}, X^{s-1}\right)}^{s-1}\right|>1$. With probability one for all $i, j \in \Psi_{i_{p}^{*}\left(v_{s}, X^{s-1}\right)}^{s-1}$ we have $X_{i}^{n}=X_{j}^{n}, \quad n=0,1, \cdots, s-1$. This can be the case only if $Y_{i}^{n}=Y_{j}^{n}$, which implies that for both $X^{s-1}$ and $Y^{s-1} \Psi_{i_{p}^{*}\left(v_{s}, X^{s-1}\right)}^{s-1}$ is the same, that is, for $j \in \Psi_{i_{p}^{*}\left(v_{s}, X^{s-1}\right)}^{s-1}$ we have $Y_{i}^{s-1}=Y_{j}^{s-1}$ iff $i \in \Psi_{i_{p}^{*}\left(v_{s}, X^{s-1}\right)}^{s-1}$. This together with $Y^{s-1} \in S\left(X^{s-1}, \eta\right)$ and $\min _{k, l \in I, X_{k}^{s-1} \neq X_{l}^{s-1}}\left|X_{k}^{s-1}-X_{l}^{s-1}\right|>6 \eta \quad$ implies that $i_{p}^{*}\left(v_{n}, Y^{n-1}\right)=i_{p}^{*}\left(v_{n}, X^{n-1}\right)$ for $n=s$.

For $\left|\Psi_{i_{p}^{*}\left(v_{s}, X^{s-1}\right)}^{s-1}\right|=1, \quad$ the definition of $i_{p}^{*}(v)$ implies $i_{p}^{*}\left(v_{s}, X^{s-1}\right)=i_{p}^{*}\left(v_{s}, Y^{s-1}\right)$.

Now equation (4) holds, that is, after the event $v_{l}$ we have $Y^{l} \in S\left(X^{l}, \eta\right)$.

We come to the conclusion that if $Y^{0}=y \in S^{p}(x, \eta) \cup S^{m+1, p}(x, \eta)$ and $\eta<$ $0.5 \min \left(\left|\bar{X}_{i_{p}^{*}+1}^{n}-v_{n}\right|,\left|\bar{X}_{i_{p}^{*}}^{n}-v_{n}\right|\right)$, then after any event $\left(v_{1}, \cdots, v_{t}\right) \in \mathcal{V}_{p}(x)$, by putting $i^{*}(v):=i_{p}^{*}(v)$, we have $Y^{t} \in S\left(X^{t}, \eta\right)$, that is, for sufficiently small $\eta, \quad Y^{t} \in F$.

Remark 1. Let $\mathcal{V}_{p}^{\alpha}(x) \subset \mathcal{V}_{p}(x)$ be the set of all events $\left(v_{1}, \cdots, v_{t}\right)$ taking any $y \in$ $S^{p}(\alpha, x) \cup S^{m+1, p}(\alpha, x)$ to $F$. We have $\mathcal{V}_{p}^{\beta}(x) \subset \mathcal{V}_{p}^{\alpha}(x)$ if $\beta>\alpha$. Furthermore from the proof of the Lemma 1 it follows that if $P$ is diffuse, then for any $\epsilon>0$ there exists an $\alpha>0$ such that $\mathbf{P}\left(\mathcal{V}_{x} \backslash \mathcal{V}_{x}^{\alpha}\right)<\epsilon$. 
Lemma 2. If $P$ is diffuse, then, for all $t \in \mathbb{N}, \quad P_{x}\left(\tau_{F} \leq t\right)$ is lower semi-continuous on $[0,1]^{N}$.

Proof. To establish the claim of Lemma we need to prove for any arbitrary sequence $x^{k}$ if $x^{k}, x \in[0,1]^{N}$ and $x^{k} \rightarrow x$ as $k \rightarrow \infty$, then $\underline{\lim }_{k \rightarrow \infty} P_{x^{k}}\left(\tau_{F} \leq t\right) \geq P_{x}\left(\tau_{F} \leq t\right)$.

Let $m$ denotes the number of ordering functions in $\Sigma_{N}^{x}$. There exists an integer $K$ such that for all $k \geq K$ we have $\Sigma_{N}^{x^{k}} \subset \Sigma_{N}^{x}$. This implies that we can split up the sequence $x^{k}, \quad k=K, \cdots$ into $m$ disjoint subsequences $x^{k, 1}, \cdots, x^{k, m}$ such that $x^{k, p} \in S^{p}(x, \eta) \cup S^{p, m+1}(x, \eta)$ for $p=1, \cdots, m$ if $\eta$ is sufficiently small .

Now Lemma 1 implies $\underline{\lim }_{k \rightarrow \infty} P_{x^{k, p}}\left(\tau_{F} \leq t\right) \geq \mathbf{P}\left(\mathcal{V}_{p}^{\alpha}(x)\right)$ for any sufficiently small $\alpha>0$, which implies $\underline{\lim }_{k \rightarrow \infty} P_{x^{k, p}}\left(\tau_{F} \leq t\right) \geq \mathbf{P}\left(\mathcal{V}_{p}(x)\right)$. On the other hand, we have $\mathbf{P}\left(\mathcal{V}_{p}(x)\right) \geq$ $P_{x}\left(\tau_{F} \leq t\right)$, which implies

$$
\underline{\lim }_{k \rightarrow \infty} P_{x^{k}}\left(\tau_{F} \leq t\right) \geq P_{x}\left(\tau_{F} \leq t\right) \quad \forall \quad x \in[0,1] .
$$

In the proof of Lemma 3 we use the known fact that for a decreasing sequence of closed sets $F_{t}$, if $\bigcap_{t \geq 0} F_{t}=\varnothing$, then there exists a $T$ such that $F_{T}=\varnothing$, see e.g. [8] .

Lemma 3. Suppose $P$ is diffuse and supp $P=[0,1]$. If $P_{x}\left(\tau_{F}<\infty\right)>0$ for all $x \in[0,1]^{N}$, then

$$
\exists \quad \theta>0, \quad \exists \quad T \in \mathbb{N} \text { such that } \forall x \in[0,1]^{N}, \quad P_{x}\left(\tau_{F} \leq T\right)>\theta .
$$

Proof. Let be $F_{t}=\left\{x \in[0,1]^{N} \quad \mid \quad P_{x}\left(\tau_{F} \leq t\right)=0\right\}$. The assumption $\quad P_{x}\left(\tau_{F}<\infty\right)>$ 0 implies that $\bigcap_{t \geq 0} F_{t}=\varnothing$. Moreover $F_{t+1} \subset F_{t}$. Using the lemma 1 we know that $F_{t}$ is closed for all $t$ and this implies the existence of $T \in \mathbb{N}$ with $F_{T}=\varnothing$. Now $P_{x}\left(\tau_{F} \leq T\right)$ is a positive and lower semi-continuous function of $x$ on $[0,1]^{N}$ which implies the existence of a lower bound $\theta>0$.

It is remarkable that the lemmas 1-3 remain true if we replace $F$ with any other open subset of $[0,1]^{N}$.

Lemma 4. Suppose $P$ is diffuse, supp $P=[0,1], \quad \mathbf{k} \geq 1, \quad \gamma_{\mathbf{j}+1}<$ $\gamma_{\mathbf{j}}$ for some $\mathbf{j}, \quad 0 \leq \mathbf{j} \leq \mathbf{k}$ and $N \geq 2 \mathbf{j}+1$. For any $x \in D^{\prime}$ there exists a $f$ nite integer $T^{\prime}$ such that $P_{x}\left(\tau_{D}<T^{\prime}\right)>0$. 
Proof. We show for any arbitrary $o^{s}(.) \in \Sigma_{N}^{x}$ there exists some positive $T^{\prime}$ such that $\mathbf{P}\left(X^{T^{\prime}} \in D \quad \mid \quad X^{0}=x \quad, i^{*}(v):=i_{s}^{*}(v)\right)>0$. Remind that $\quad \omega_{i}:=\{i-\mathbf{j}, \cdots, i, \cdots, i+$ j\} $\cap I$.

Suppose $\left|\Psi_{i}^{0}\right| \leq l, \quad l>1$, for all $i \in I$. We show that $\left|\Psi_{i}^{R}\right| \leq l-1$ for all $i \in I$ with a positive probability, where $R \leq 3|A| / l \quad$ and $\quad A:=\left\{i \in I|\quad| \Psi_{i}^{0} \mid=l\right\}$.

Let $\left|\Psi_{i}^{0}\right|=l$ and $p, q$ be the smallest and greatest orderings in $\Psi_{i}^{0}$ according to $o^{s}(.) \in \Sigma_{N}^{x}$, respectively.

If either $\Psi_{i}^{0} \backslash \omega_{o^{s}(q)} \neq \varnothing$ or $\Psi_{i}^{0} \backslash \omega_{o^{s}(p)} \neq \varnothing$, then the event $i^{*}\left(v_{1}\right)=o^{s}(q)$ or $i^{*}\left(v_{1}\right)=o^{s}(p)$ leads to $\Psi_{i}^{1} \leq l-1$, respectively. Both of these events may happen with a positive probability.

Now suppose $\Psi_{i}^{0} \subset \omega_{o^{s}(q)}$ and $\Psi_{i}^{0} \subset \omega_{o^{s}(p)}$. This can happen only if $l \leq 2 \mathbf{j}$. There exists a neuron $u$ such that $u \notin \Psi_{i}^{0}$ and either $u \in \omega_{o^{s}(q)} \backslash \omega_{o^{s}(p)}$ or $u \in \omega_{o^{s}(p)} \backslash \omega_{o^{s}(q)}$. Without loss of generality, we suppose $u \in \omega_{o^{s}(q)} \backslash \omega_{o^{s}(p)}$.

If the event $i^{*}\left(v_{1}\right)=u$ happens with a positive probability, then the probability of $\left|\Psi_{i}^{1}\right| \leq l-1$ is positive,too. In otherwise, after the events $i^{*}\left(v_{1}\right)=o^{s}(q)$ and $i^{*}\left(v_{2}\right)=o^{s}(p)$ we have $\Psi_{u}^{1} \subset \omega_{o^{s}(q)}$ and $\Psi_{u}^{2} \cap \omega_{o^{s}(p)}=\varnothing$. Let $q^{\prime}$ be the greatest ordering acording to $o^{s}($.$) in \Psi_{u}^{2}$. Then the event $i^{*}\left(v_{3}\right)=o^{s}\left(q^{\prime}\right)$ moves $o^{s}(q)$ while $o^{s}(p)$ remains fixed, i.e., with a positive probability we have $\left|\Psi_{i}^{3}\right| \leq l-1$.

Repeating these events for all different set of neurons with $\left|\Psi_{i}^{0}\right|=l$, after $R$ steps we have $\left|\Psi_{i}^{R}\right| \leq l-1$ for all $i$.

Theorem 1. Suppose $P$ is diffuse, supp $P=[0,1], \quad \Sigma_{n} \epsilon_{n}=\infty, \quad \mathbf{k} \geq 1, \quad \gamma_{\mathbf{j}+1}<$ $\gamma_{\mathbf{j}}$ for some $\mathbf{j}, \quad 0 \leq \mathbf{j} \leq \mathbf{k}$ and $N \geq 2 \mathbf{j}+1$. Then

$$
\exists \quad \theta>0 \quad \exists \quad T \in \mathbb{N} \text { such that } \forall x \in[0,1]^{N}, \quad P_{x}\left(\tau_{F} \leq T\right)>\theta .
$$

Proof. To establish the assertion using Lemmas 3 and 4 and the Markov property it is enough to show that for any $x \in D$ there exists a $T^{\prime \prime} \in N$ such that $\mathbf{P}\left(\tau_{F} \leq T^{\prime \prime} \mid X^{0}=\right.$ $x)>0$. We will introduce a finite chain of events which takes $X^{0}=x \in D$ to $F$ with a positive probability. We emphasize that there are other chain of events which do the job as well.

Note that if $X^{n} \in D$, then every neuron may win at time $n$ with a positive probability.

Let $F_{k}^{+}=\left\{x \in[0,1]^{N} \quad \mid \quad 0<x_{1}<\cdots<x_{k}, \quad x_{i}>x_{k} \quad \forall \quad i>k\right\}$.

If $x \notin F_{1}^{+}$but there exists a neuron $r$, with $r>\mathbf{j}+1$ such that $x_{r}>x_{1}$ then after the event

$$
i^{*}\left(v_{n}\right)=r, \quad 1 \leq n<T_{1}
$$


for sufficiently big $T_{1}$ we have $x_{i}>x_{1}$ for $i \in \omega_{r}$. With an analogous argument the following chain of events takes $x$ to $F_{1}^{+}$:

$$
\begin{aligned}
i^{*}\left(v_{n}\right)=r+\mathbf{j}, & T_{1} \leq n<T_{2}, \\
\vdots & \\
i^{*}\left(v_{n}\right)=r+l \mathbf{j}, & T_{l-1} \leq n<T_{l}, \\
i^{*}\left(v_{n}\right)=r-\mathbf{j}, & T_{l} \leq n<T_{l+1}, \\
\vdots & \\
i^{*}\left(v_{n}\right)=r-p \mathbf{j}, & T_{l+p-1} \leq n<T_{l+p}, \\
i^{*}\left(v_{n}\right)=\mathbf{j}+2, & T_{l+p} \leq n<T_{l+p+1}
\end{aligned}
$$

Here $r+l \mathbf{j} \leq N<r+(l+1) \mathbf{j}$ and $r-(p+1) \mathbf{j}<\mathbf{j}+2 \leq r-p \mathbf{j}$. The times $T_{s}, \quad 1 \leq$ $s \leq l+p+1$, are a numbers of iterations after which we have $x_{i}>x_{1}$ for all $i \in$ $\omega_{i^{*}\left(v_{T_{s-1}}\right)}$. The condition $\quad \Sigma_{n} \epsilon_{n}=\infty$ implies that $T_{s}$ is finite for all $1 \leq s \leq l+p+1$. If $x \in F_{k}^{+} \quad$ for some $k \in\{1, \cdots, \mathbf{j}-1\}$, then after the chain of events

$$
i^{*}\left(v_{n}\right)=k, \quad 1 \leq n<T_{1}^{\prime},
$$

we have $\quad x \in F_{k}^{+}$and moreover $x_{k}<x_{i}<x_{l}$ for all $i \in\{k+1, \cdots, k+\mathbf{j}\}$ and $l \geq k+\mathbf{j}+1$.

Now the following chain of events takes $x$ to $F_{k+1}^{+}$:

$$
i^{*}\left(v_{n}\right)=k+\mathbf{j}+2, \quad T_{1}^{\prime} \leq n<T_{2}^{\prime},
$$

where $T_{2}^{\prime}$ is the smallest time after which we have $x_{k+1}<x_{i}$ for all $k+1<i \leq N$. Again $\Sigma_{n} \epsilon_{n}=\infty$ implies that $T_{1}^{\prime}$ and $T_{1}^{\prime}$ are finite.

If $x \in F_{k}^{+}$and $\quad k \geq \mathbf{j}$ then the event $i^{*}\left(v_{n}\right)=k-\mathbf{j}+1$ moves the neuron $k+1$ to the left while the neurons $k+2$ up to $N$ remain fixed. Repeating this event after some finite steps $T_{1}^{\prime \prime} \in N$ we have $x_{k+1}<x_{i}$ for all $i>k+1$, that is, $X^{T_{1}^{\prime \prime} \in F_{k+1}^{+}}$.

The only case which remains is $x \notin F_{1}$ and there does not exists some neuron $r>$ $\mathbf{j}+1$, such that $x_{r}>x_{1}$. In this case with exactly the same argument, this time for $F_{k}^{-}:=\left\{x \in[0,1]^{N} \quad \mid \quad 1>x_{1}>\cdots>x_{k}, \quad x_{i}<x_{k} \quad \forall i>k\right\}$, it can be shown that after a finite number of iterations with a positive probability $X^{\mathcal{T}} \in F_{1}^{-}$. Moreover if $x \in F_{k}^{-} \quad 1 \leq k<N$, then with a positive probability after some finite time we have $X^{\mathcal{T}^{\prime}} \in F_{k+1}^{-}$.

It should be mentioned that, the constant $\theta$ generally is time dependent, so that the Theorem 1 can not be considered as a with probability one self-organization result. Nevertheless, in case of a constant learning rate, $\theta$ is time independent and the following extension of the Theorem 1 holds. 
Theorem 2. Suppose $P$ is diffuse, supp $P=[0,1], \quad \epsilon_{n}$ is constant, $\mathbf{k} \geq$ $1, \quad \gamma_{\mathbf{j}+1}<\gamma_{\mathbf{j}}$ for some $\mathbf{j}, \quad 0 \leq \mathbf{j} \leq \mathbf{k}$ and $N \geq 2 \mathbf{j}+1$. Then there exists a finite integer $T$ such that for all $x \in[0,1]^{N}$ and $o^{k}(.) \in \Sigma_{N}$ we have

$$
\mathbf{P}\left(\tau_{F} \leq T \mid X^{0}=x, i^{*}(v):=i_{k}^{*}(v)\right)=1 .
$$

Remark 2. To establish the Theorems 1 and 2 we did not used the symmetry of the neighborhood function $f$. Therefore these results are also valid for asymmetric nonincreasing neighborhood functions. In such a cases $\mathbf{j}$ is the smallest index which satisfies $\gamma_{\mathbf{j}+1}<1$ and $\gamma_{-\mathbf{j}-1}<1$.

\section{Conclusion}

Here we proved the almost sure self-organization of one-dimensional Kohonen algorithm, under quite reasonable restrictions on the stimuli distribution, neighborhood function and the learning rate. A natural question which arises is how to generalize the proof to the case of a more general data space and network structure? There are two major difficulties which prevent such a generalization.

Firstly, there does not exists an absorbing set like $F$ in multi-dimensional cases. Nevertheless, the set of organized states may be defined, see $[5,7,12]$ for different definitions. A possible approach to tackle the multi-dimensional case could be to show a weak self-organization property, in the sense that, the neuron weights go to an organized configuration in finite time with probability one, although later on they may leave this configuration.

Secondly, no generalization of winner definition is known, which possesses the properties of the $k$-winner as was defined in section 2 of this paper. It was the only difficulty which prevented the establishment of a result similar to Lemmas 1-3 for multi-dimensional algorithm. 


\section{NOMENCLATURE}

F

$F^{+}$

$F^{-}$

$f_{i i^{*}}$

$I$

j

k

$\underline{\lim }$

$n$

$N$

$o^{k}($.

$P$

$\mathbf{P}(. \mid$.

$Q$

$\mathbb{R}$

$\mathbb{N}$

$X^{n}$

$|\Psi|$

$\tau_{\pi}$

$\epsilon_{n}$

$\gamma_{i}$

$\varnothing$

$\omega_{i}$
Set of organized configurations

Set of ascending configurations

Set of descending configurations

Neighborhood function

Set of neurons

The smallest index with $\gamma_{\mathbf{j}}<1$.

Width of neighborhood function

Lower limit point of a sequence

Discrete time index, $n \in \mathbb{N}$

Total number of neurons

Ordering function

Data distribution

The conditional probability function

Data space

Set of real numbers

Set of positive integers numbers

Weight vector at time $n$

Number of the elements of a set $\Psi$

The time to reach a set $\pi$

Learning rate

Neighborhood function for the $i$ th neuron

Empty set

$\{i-\mathbf{j}, \cdots, i, \cdots, i+\mathbf{j}\} \cap I$

\section{References}

[1] Bouton, C., \& Pagés, G. (1993) Self-organization and a.s. convergence of the onedimensional Kohonen algorithm with non-uniformly distributed stimuli.Stochastic Procces and their Applications , 47, 249-274.

[2] Bouton, C., \& Pagés, G. (1994) Convergence in distribution of the one-dimensional Kohonen algorithm when the stimuli are not uniform . Advances in Applied Probability, 26, 80-103 .

[3] Cottrell,M., \& Fort,J.C. (1987). Étude d'un processus d'auto-organisation . Annales de l'Institut Henri Poincaré,23(1) , 1-20 (in French).

[4] Erwin, E., Obermeyer,K. \& Schulten ,K.(1992). Self-organizing maps: ordering, convergence properties and energy functions.Biological Cybernetic ,67 , 47-55 .

[5] Flanagan,J.A. (1996). Self-organisation in Kohonen's SOM,Neural Networks ,7 , 11851197. 
[6] Flanagan,J.A. (1997). Self-organisation in the one -dimensional SOM with a reduced width neighbourhood,Proc. SOM Workshop, Helsinki.

[7] Fort, J.C., Pagés, G. (1996) . About the Kohonen Algorithm: Strong or Weak SelfOrganization ? Neural Networks, 5, 773-785.

[8] Hewitt, E. \& Stromberg, K. (1969).Real and abstract analysis. Springer-Verlag .

[9] Kohonen, T. (1982). Self-organized formation of topologically correct feature maps.Biological Cybernetics,43, 59-69.

[10] Kohonen,T. (1995). Self-organizing maps, Springer-Verlag .

[11] Sadeghi, A.A. . Asymptotic behavior of self-organizing maps with non-uniform stimuli distribution, preprint 166, Arbeitsgruppe Technomathematik, Universität Kaiserslautern, Juli 1996, (to appear in The Annals of Applied Probability).

[12] Villmann,T., Der, R. , M. Herrmann, M. \& Martinetz,T. .(1997). Topology preservation in self-organizing feature maps: Exact definition and measurement.IEEE Transaction on Neural Networks, 2, , 256-266. 